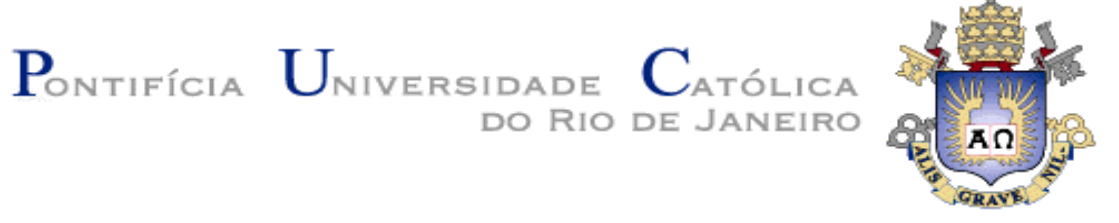

Joaquim Ignacio Baptista Cardoso Filho

\title{
Quantificação de Ni e V em frações polares de óleo bruto com o uso de cromatografia líquida e ICPMS
}

Dissertação de Mestrado

Dissertação apresentada ao Programa de Pós-Graduação em Química da PUC-Rio como requisito parcial para a obtenção do título de Mestre em Química .

Orientador: Prof. Norbert Miekeley

Co-Orientadora: Prof. Christiane Béatrice Duyck

Rio de Janeiro

Setembro de 2009 


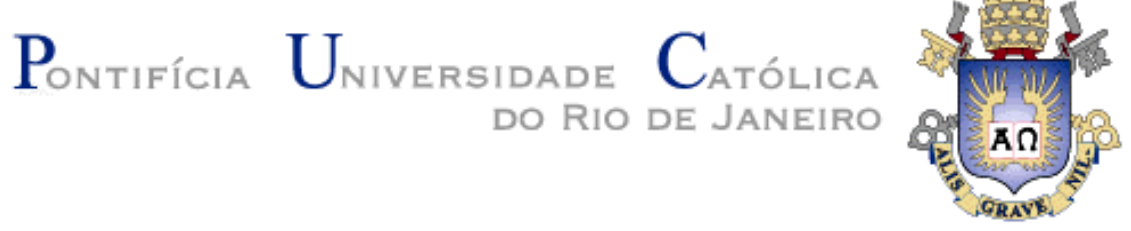

Joaquim Ignácio Baptista Cardoso Filho

\section{Quantificação de Ni e V em frações polares de óleo bruto com o uso de cromatografia líquida e ICPMS}

Dissertação apresentada como requisito parcial para obtenção do grau de Mestre pelo Programa de Pós-Graduação em Química da PUC-Rio. Aprovada pela Comissão Examinadora abaixo assinada.

Prof. Norbert Miekeley

Orientador

Departamento de Química - PUC-Rio

Prof. Christiane Béatrice Duyck

Co-Orientadora

Departamento de Química - PUC-Rio

Dra. Teresa Cristina Oliveira Fonseca

CENPES - Petrobras

Prof. Tatiana Dillenburg Saint'Pierre

Departamento de Química - PUC-Rio

Prof. José Eugenio Leal

Coordenador Setorial do Centro

Técnico Científico - PUC - Rio

Rio de Janeiro, 25 de setembro de 2009 
Todos os direitos reservados. É proibida a reprodução total ou parcial do trabalho sem autorização da universidade, do autor e do orientador.

Joaquim Ignácio Baptista Cardoso Filho Graduou-se em Química na UFF (Universidade Federal Fluminense) em 2006. Cursou Mestrado em química analítica na PUC-Rio entre 2007 e 2009. Trabalha na área de análise instrumental com ênfase em química do petróleo

Ficha Catalográfica

Cardoso Filho, Joaquim Ignacio Baptista

Quantificação de NI e V em frações polares de óleo bruto com o uso de cromatrografia líquida e ICPMS I Joaquim Ignacio Baptista Cardoso Filho ; orientador: Norbert Miekeley ; co-orientadora: Christiane Béatrice Duyck. - 2009.

77 f. : il. (color.) ; $29,7 \mathrm{~cm}$

1. Dissertação (Mestrado em Química)Pontifícia Universidade Católica do Rio de Janeiro, Rio de Janeiro, 2009.

Inclui bibliografia

1. Química - Teses. 2. ICPMS. 3. Cromatografia líquida. 5. Porfirinas. I. Miekeley, Norbert. II. Duyck, Christiane Béatrice. III. Pontifícia Universidade Católica do Rio de Janeiro. Departamento de Química. IV. Título. 


\section{Agradecimentos}

A Deus, que me protege todos os dias e me dá forças para prosseguir em meus objetivos.

A minha Mãe Mariceli, por tudo que fez e que faz por mim, pelo amor e pelo apoio incondicional que dá para tudo que faço.

A minha noiva, Elizabeth, por todo o amor e companheirismo que me deu durante o período de realização deste trabalho, sendo compreensiva comigo em relação aos momentos em que não pude estar com ela.

Ao Professor Norbert Miekeley, pela orientação e a oportunidade de realizar este trabalho.

Á doutora Christiane Béatrice Duyck, pela dedicação e orientação dadas durante o trabalho. sem sua presença constante, esse trabalho não teria sido possível. Muito obrigado.

Aos técnicos do LABSPECTRO Àlvaro Pereira, Danielle Gonçalves e Rafael Cristian, por todo o apoio técnico prestado e pelo modo atencioso com que me atenderam sempre que eu necessitei.

A todos os funcionários da área de apoio e administrativa do LABSPECTRO, em especial a Adriana Silva, por toda ajuda que me foi dispensada quando necessitei. 
Aos membros do laboratório de estudos ambientais e toxicologia (LEATOX) pela permissão e auxílio no uso do espectrofotômetro de UV-Vis.

Aos funcionários da secretaria de pós-graduação, em especial a Fátima Almeida.

A todos os professores que lecionaram matérias das quais fui aluno na pósgraduação, pelos conhecimentos que me foram passados.

A todos os colegas e amigos que fiz nessa pós-graduação, em especial para Cibele Stivanin, pelos ótimos momentos de convivência.

A meus amigos e "irmãos" de infância, Moreno e Rodrigo por toda amizade e momentos de risadas dadas comigo a durante o tempo de duração do trabalho, mesmo sem entender o que eu fazia, me apoiaram sempre a prosseguir.

Á PETROBRAS petróleo brasileiro S.A, pelo apoio financeiro dado ao projeto de pesquisa deste trabalho.

Ao $\mathrm{CNPq}$, órgão financiador da bolsa, essencial para a minha permanência e continuidade dos estudos. 


\section{Resumo}

Filho, Joaquim Ignácio Baptista Cardoso; Miekeley, Nobert; Duyck, Christiane Béatrice. Quantificação de Ni e V em frações polares de óleo bruto com o uso de cromatografia líquida e ICPMS. Rio de Janeiro, 2009. 77p Dissertação de Mestrado - Departamento de Química, Pontifícia Universidade Católica do Rio de Janeiro

Ni e V ocorrem em frações polares de óleos brutos e são de interesse geoquímico. Um método foi desenvolvido para isolar e quantificar esses metais associados a compostos orgânicos chamados porfirinas, usando-se cromatografia líquida e espectrometria de massa com plasma indutivamente acoplado (ICPMS). A fração do óleo contendo parafinas, aromáticos e polares foi separada usando-se uma coluna de vidro de $25 \mathrm{~cm}$ x 2,5 cm preenchida com sílica gel, empregando-se um programa automatizado de gradiente de eluição com ordem crescente da polaridade dos solventes. O eluido foi analisado por espectrofotometria UV-vis e o cromatograma obtido permitiu a distinção de três frações, que foram analisadas separadamente por ICPMS. O sistema de introdução de amostra do ICPMS consistiu em um micronebulizador concêntrico de Teflon acoplado a uma câmara refrigerada a $-14{ }^{\circ} \mathrm{C}$. Oxigênio foi introduzido no gás de nebulização (Ar) para evitar a formação de carbono na interface do instrumento. As condições operacionais foram otimizadas por planejamento experimental. A vazão de Ar foi de $0,46 \mathrm{~L} \mathrm{~min}^{-1}$ e a de $\mathrm{O}_{2}$ foi de $0,1 \mathrm{~L} \mathrm{~min}^{-1}$, a $1350 \mathrm{~W}$. Os limites de detecção para o $\mathrm{V}$ e o $\mathrm{Ni}$ foram de 29 e $465 \mathrm{ng} \mathrm{L}^{-1}$ respectivamente. 
A concentração de Ni foi de $(7,1 \pm 0,8),(16,8 \pm 1,6)$ e $(12 \pm 2) \mu \mathrm{g} \mathrm{g}^{-1}$ para as frações 1,2 e 3 respectivamente. $\mathrm{O} \mathrm{V}$ foi quase que exclusivamente encontrado na terceira fração (mais polar) com $(30 \pm 3) \mu \mathrm{g} \mathrm{g}^{-1}$. Esses resultados sugerem a associação do V com frações mais pesadas do óleo.

\section{Palavras-chave}

ICPMS; cromatografia líquida; porfirinas 


\section{Abstract}

Filho, Joaquim Ignácio Baptista Cardoso; Miekeley, Nobert (Advisor); Duyck, Christiane Béatrice (Co-advisor). Quantification of $\mathrm{Ni}$ and $\mathrm{V}$ in polar fractions of crude oil using liquid chromatography and ICPMS Rio de Janeiro, 2009. 77p MSc Dissertation - Departamento de Química, Pontifícia Universidade Católica do Rio de Janeiro.

$\mathrm{Ni}$ and $\mathrm{V}$ occur in the polar fraction of crude oils and have geochemical interest. A method was developed for isolating and quantifying these metals associated with organic compounds like porphyrins, using liquid chromatography and inductively coupled plasma mass spectrometry (ICPMS). The oil fraction containing paraffins, aromatics and polars was separated using a glass column of $25 \mathrm{~cm} \times 2.5 \mathrm{~cm}$ filled with silica gel. An automatic elution program was used to create a solvent elution gradient of increasing polarity. The column eluate were analyzed by UV-vis spectrophotometry and the chromatograms obtained showed the separation of three fractions, which were then analyzed separately by ICPMS. The ICPMS sample introduction system consisted of a concentric Teflon micronebulizer coupled to a refrigerated spray chamber set to $-14{ }^{\circ} \mathrm{C}$. Oxygen was introduced into the nebulization gas flow to prevent de formation of carbon at the instrument interface. Operational conditions were optimized by experimental design. The optimum gas flow rates were $0.46 \mathrm{~L} \mathrm{~min}^{-1}$ (Ar) and $0.1 \mathrm{~L} \mathrm{~min}^{-1}\left(\mathrm{O}_{2}\right)$, at $1350 \mathrm{~W}$. 
The detection limits for $\mathrm{V}$ and $\mathrm{Ni}$ were 29 and $465 \mathrm{ng} \mathrm{\textrm {L } ^ { - 1 }}$, respectively. Ni concentrations in each fraction were $(7,1 \pm 0,8),(16,8 \pm 1,6)$ and $(12 \pm 2) \mu \mathrm{g} \mathrm{g}^{-1}$, for the fractions 1, 2 and 3 respectively.V was almost exclusively concentrated in the third (most polar) fraction at $(30 \pm 3) \mu \mathrm{g} \mathrm{g}^{-1}$. These results suggest that $\mathrm{V}$ is associated with the heavier fractions of the oil.

\section{Keywords}

ICPMS; liquid chromatography; porphyrins 


\section{Sumário}

1 Introducão e objetivos

1.1 Ocorrência de Ni e $\mathrm{V}$ nas frações de aromáticos e polares

de óleo bruto.

2.2 Determinação de Ni e $\mathrm{V}$ em óleo e derivados por ICPMS

após solubilização em solventes

2 Materiais e metodologias

2.1 separação cromatografica

2.1.1 Precipitação de asfaltenos

28

2.1.2 Fracionamento dos maltenos por cromatografia líquida $(C L)$

2.1.2.1. Separação de parafinas, aromáticos e polares (PAP)

a partir dos maltenos

2.1.2.2. Fracionamento por cromatografia líquida com bombeamento de solvente

2.1.2.3. Sistema de cromatografia líquida automatizado

2.1.3 Detecção por espectrofotometria de UV-Vis

2.2 Análise no ICPMS após diluição em tolueno. 
2.2.2 Planejamento experimental dos parâmetros físicos

2.2.3 Parâmetros operacionais. $\quad 40$

2.2.4 Determinação de Ni e $\vee$ em frações de maltenos.

3 Resultados e discussões 43

3.1 Separação de subfrações porfirínicas $\quad 43$

3.1.1 Fracionamento de maltenos 43

3.1.2 Separação automatizada $\quad 44$

3.2 Análise por ICPMS após solubilização das amostras em tolueno $\quad 50$

3.2.1 Otimização das condições analíticas $\quad 50$

3.2.1.1 Avaliação da importância dos fatores experimentais 50

3.2.1.2 Otimização do micronebulizador com álcoois 53

3.2.1.3 Condições de uso do micronebulizador com tolueno 54

3.2.2 Curvas de calibração e parâmetros de mérito analítico 55

3.3 Resultados de V e Ni em subfrações de maltenos do óleo T1 61

4 Conclusões $\quad 65$

$\begin{array}{ll}5 \text { Referências bibliográficas } & 67\end{array}$

6 Anexos $\quad 73$ 


\section{Lista de figuras}

Figura 1.1: Porfirinas de V: a) DPEP e b) Etio 20

Figura 2.1: Esquema do fracionamento do óleo bruto dessalinizado. 26

Figura 2.2: Sistema de cromatografia líquida em coluna de vidro (C) com bomba peristáltica (P): (1) banda de eluição; (2) saída da banda de eluição e recuperação do eluido em cortes (frações) de $3 \mathrm{~mL}$

Figura 2.3: Sistema automatizado de cromatografia líquida por bombeamento de solvente (LC 200, PE-Sciex), com válvula quaternária $(\mathrm{V})$. DCM = diclorometano. 31

Figura 2.4: ELAN 6000 com o sistema de introdução empregado 33

Figura 2.5: Micronebulizador PFA-100. 34

Figura 2.6: Sistema Isomist ${ }^{\mathrm{TM}}$ indicando o ponto de entrada de $\mathrm{O}_{2}$. 34 Figura 3.1: Testes A e B mostrando a separação de maltenos por cromatografia líquida em coluna de silicagel com vazão variável da fase móvel $\left(0,5\right.$ a $\left.1,5 \mathrm{~mL} \mathrm{~min}{ }^{-1}\right)$.

Figura 3.2: Separação da fração de parafinas, aromáticos e polares (Separação II) em 400 nm e 500 nm (A) e 400 nm e 570 nm (B).

Figura 3.3: Espectro de UV-Vis de varredura entre 350 a $700 \mathrm{~nm}$ do padrão NiOEP com concentração de1 $\mathrm{mg} \mathrm{L}^{-1}$.

Figura 3.4: Espectro de UV-Vis de varredura entre 350 e $700 \mathrm{~nm}$ do padrão NiEtioP com concentração de1 $\mathrm{mg} \mathrm{L}^{-1}$.

Figura 3.5: Espectro de UV-Vis de varredura entre 350 e $700 \mathrm{~nm}$ do padrão VO-OEP com concentração $1 \mathrm{mg} \mathrm{L}^{-1}$.

Figura 3.6: Espectros típicos de absorção de eluidos com varredura de 350 a $700 \mathrm{~nm}$, nos intervalos 85-120 mL (A) e 160-215 mL (B). 
Figura 3.7: Gráficos de Pareto mostrando os efeitos de sete fatores

na intensidade de $\mathrm{Rh}$ e nas razões das intensidades $\mathrm{Ba}^{2+} / \mathrm{Ba}$ e $\mathrm{CeO}^{+} / \mathrm{Ce} . \quad 52$

Figura 3.8: Variação de log (++ / O+)em função da vazão

de $\operatorname{Ar}\left(L \min ^{-1}\right)$

Figura 3.9: Gráficos de superfície de resposta para

o $\ln (\mathrm{A}), \mathrm{Ba}^{2+}(\mathrm{B})$ e $\mathrm{Pb}(\mathrm{C})$. $\quad 56$

Figura 3.10: Gráficos de superfície de resposta para o V. 57

Figura 3.11: Gráficos de superfície de resposta para o Ni. 57

Figura 3.12: Curvas de calibração para o V-51, Ni-58 e Ni-60

em solução de tolueno.

58

Figura 3.13 : Distribuição de Ni e V nas subfrações resultantes

da separação dos maltenos do óleo T1 utilizados no teste $B$.

Figura 3.14: Distribuição de Ni e V nas subfrações F1, F2 e F3

da separação da fração de PAP do óleo T1.

Figura 6.1: Curva de absorvância x concentração do padrão

NiOEP em $550 \mathrm{~nm}$.

Figura 6.2: Curva de absorvância x concentração do padrão

NietioP em $550 \mathrm{~nm}$.

74

Figura 6.3: Curva de absorvância x concentração do padrão VOEP em $550 \mathrm{~nm}$.

Figura 6.4: Curva de absorvância x concentração do padrão NiOEP em $570 \mathrm{~nm}$.

Figura 6.5: Curva de absorvância x concentração do padrão

NiEtioP em $570 \mathrm{~nm}$.

Figura 6.6: Curva de absorvância x concentração do padrão 


\section{Lista de tabelas}

Tabela 2.1 Composição, parâmetros físico-químicos e concentração de $\mathrm{V}$ e Ni (mg kg-1) no óleo, maltenos, asfaltenos, fração de aromáticos e polares (aro+pol), e na resina do óleo $\mathrm{T} 1$.

Tabela 2.2 Alguns dados físico-químicos dos solventes empregados neste estudo

Tabela 2.3 Parâmetros de aquisição de dados no ICPMS para o planejamento experimental

Tabela 2.4 Parâmetros estudados do ICPMS com

Tabela 2.5 Experimentos do planejamento $2^{(7-4)}$

Tabela 2.8 Valores de alguns parâmetros operacionais do ICPMS

Tabela 3.1: Resultados obtidos do óleo T1 para as separações I e II.

Tabela 3.2: Resultados obtidos para as separações da PAP do malteno (óleo T1). 
Tabela 3.3: Absortividades (em $\mathrm{L} \mathrm{mol}^{-1} \mathrm{~nm}^{-1}$ )

Dos padrões em diferentes comprimentos de onda.

Tabela 3.4: Parâmetros de mérito analítico para o V-51, Ni-58 e Ni-60.

Tabela 3.5: Concentrações médias, desvios padrão

(DP para $n=6$, DPR entre parênteses) e recuperações

médias para o padrão NIST 1085b com concentração

de 6,0 e $60 \mathrm{ng} \mathrm{mL}^{-1}$

Tabela 3.6: Valores médios de concentração $\left(\mathrm{mg} \mathrm{kg}^{-1}\right)$

e DP $(n=3)$ para $\vee$ e Ni nas subfrações do malteno

(óleo T1) utilizado no teste B.

Tabela 3.7: Concentrações médias $\left(\mathrm{mg} \mathrm{kg}^{-1} \pm \mathrm{DP} ; \mathrm{n}=3\right.$ )

de $\mathrm{V}$ e Ni nas subfrações $\mathrm{F} 1$, F2 e F3 e na fração de PAP do óleo T1.

Tabela 6.1 Planejamento experimental $2^{(7-4)} \mathrm{com}$ metanol no nebulizador concêntrico.

Tabela 6.2 Otimização de análise com metanol no micronebulizador PFA-100

Tabela 6.3 Planejamento experimental $2^{2}$ com Tolueno no micronebulizador PFA-100 


\section{Lista de abreviaturas}

A - Absorvância, definida pela equação da lei de Lambert-Beer: $A=$ c.b.C onde: A, absorvância, b, distância percorrida pelo feixe de luz no corpo $(\mathrm{cm}), \varepsilon$, absortividade molar $\left(\mathrm{L} \mathrm{mol}^{-1} \mathrm{~cm}^{-1}\right)$ e $\mathrm{C}$, concentração $\left(\mathrm{mol} \mathrm{L}^{-1}\right)$.

CLAE ou HPLC- Cromatografia Líquida de alta eficiência (de alto desempenho).

CL- Cromatrografia Líquida.

CC- Célula de reação.

DCM - Diclorometano.

DIN- Nebulizador de injeção direta.

DIHEN- Nebulizador de injeção direta de alta eficiência.

DP- Desvio padrão, definido pela equação abaixo

$$
s=\sqrt{\frac{1}{n-1} \sum_{i=1}^{n}\left(x_{i}-\bar{x}\right)^{2}}
$$

Onde: $n$, número de replicatas, $x_{i}$, valor da replicata $\mathrm{i}, \bar{x}$, média dos valores das replicatas.

DPR- desvio padrão relativo, definido como $(\operatorname{Dp} \times 100 / \bar{x})$.

DRC- Célula de reação dinâmica

ICPMS - Espectrometria de massas com plasma indutivamente acoplado.

ICPOES- Espectrometria de emissão óptica com plasma indutivamente acoplado

LD- Limite de detecção, definido como $3 \times \sigma_{\mathrm{B}} / \mathrm{S}$ 
sendo $\sigma_{\mathrm{B}}$, o desvio padrão de 10 medidas do branco e $\mathrm{S}$, a sensibilidade do elemento, expressa pelo coeficiente angular (inclinação) de sua curva de calibração.

LQ- Limite de Quantificação, definido como $10 \times \sigma_{\mathrm{B}} / \mathrm{S}$

sendo $\sigma_{\mathrm{B}}$, o desvio padrão de 10 medidas do branco e $\mathrm{S}$, a sensibilidade do elemento, expressa pelo coeficiente angular (inclinação) de sua curva de calibração.

NietioP- Etioporfirina de $\mathrm{Ni}$

NiOEP- Octaetilporfina de níquel

NUS- nebulizador ultrassônico

PAP- Fração de parafinas, aromáticos e polares

RF- Radio freqüência.

SRM- material de referência padrão

VOEP- Octaetilporfirina de vanadil

UV-vis- Espectrofotometria na região do UV-vis. 